\title{
Image Transmission over Space Time Coded MIMO- OFDM System with Punctured Turbo Codes
}

\author{
Bagadi K. Praveen \\ Ph. D Scholar \\ Dept. of Electrical Engg. \\ NIT Rourkela, India
}

\author{
Susmita Das \\ Associate Professor \\ Dept. of Electrical Engg. \\ NIT Rourkela, India
}

\author{
Sridhar K. \\ M. Tech Student \\ Dept. of Electrical Engg. \\ NIT Rourkela, India
}

\begin{abstract}
A robust progressive compressed image using Punctured Turbo Codes (PTC) is considered while the image is transmitting over Space Time Coded (STC) Orthogonal Frequency Division Multiplexed (OFDM) systems. The Set Partitioning in Hierarchical Trees (SPIHT) technique is used regarding to image coding and compression. SPIHT compression technique fits to be best in this scheme for quality improvement. The BER performance of the PTC over Gaussian noisy channel is first evaluated by assuming perfect channel state information at the receiver for coherent detection. Then this PTC applied to end to end system over wireless fading channel. In simulation results, BER performance of PTC SPIHT system evaluated and Punctured Convolutional Coded (PCC) scheme compared with PTC scheme. Generally, Turbo codes stands to combat the interference of the fading channel. Thus, the simulation results show the effectiveness of our proposed practical image transmission system.
\end{abstract}

\section{Keywords}

OFDM, Space Time Coding, MIMO, Turbo Codes, Image Transmission, SPIHT Coding.

\section{INTRODUCTION}

Generally, a gray scale image is a two dimensional data set using 8-bit data to represent each pixel. Eventually, an image consists of huge data and transmitting this data over wireless medium is a challenging task. Hence, there is a necessity for image compression to reduce the data. This compressed image is always interfered by channel noise and these noises often happen randomly and suddenly on all the information bits. In order to overcome channel interference, efficient error correcting coding is necessary. The suitable robust channel coding and transmission systems design compensates at the cost of significant additional complexity. The specific application of image transmission over wireless channels poses a challenging research problem requiring among others, appropriate selection of the set of channel codes for effective Forward Error Correction (FEC) Codes. Due to systematic form of turbo codes, immediate extraction and decoding of source information from the channel coded stream is possible whenever the stream is not corrupted. A variety of error of resilient techniques has been recently proposed in several literatures. More are based on the state of the art set partitioning in hierarchical trees (SPIHT) [9] source codes, which generates embedded bit streams i.e., streams in which lower rates are prefixes of higher rates. In [2], a traditional approach was formulated for the protection of SPIHT streams using product codes that consists of concatenated rate compatible Punctured Convolutional Codes/CRC [3, 4]. The resulting scheme was shown to perform well for image transmission over wireless and memory less channels. Some extensive works were done using adaptive modulation and Joint Source Channel Matching (JSCM) and they are reported in $[2,5,16]$. In [7], performance of Turbo codes evaluated in Gaussian noisy channels with unequal error protection (UEP). This punctured Turbo codes [14] utilization in SPIHT [9] coded image transmission over apace time coded OFDM systems approach admits a fast dynamic programming implementation.

In this paper, a novel methodology for the transmission of images over wireless channels is proposed. Our scheme, termed Punctured turbo coded SPIHT (PTCS), was implemented over OFDM systems with spatial diversity is proposed, where neither feedback channel nor Channel State Information (CSI) is available at transmitter. OFDM [8] is an attractive technique providing high data rate and deals with frequency-selective, time dispersive wireless fading channels. Our scheme is based on previous works reported in $[1,5,16]$. Diversity techniques, including spatial, frequency, and time domain diversity, have been suggested to decrease the fading effect. Sufficiently spaced antennas are an attractive source of diversity since they do not typically incur bandwidth expansion as in frequency division diversity, and does not incur delays as in time diversity. Though spatial diversity can be available at both transmitter and receiver, it may not be possible to obtain much diversity gain at mobile terminal due to the space and power limitations at mobile terminals. We consider using space-time block codes (STBC) as a spatial diversity technique to decrease the fading effects in both time and frequency domain for OFDM systems [3, 6]. Then, a PTC scheme for OFDM system with STBC is presented for SPIHT encoded compressed image transmission for compression and error protection, where the channel state information is not available at the transmitter but known at the receiver. Further this scheme is compared to classical transmission scheme with PCC [3].

This paper is arranged as follows. In Section 2, describes the proposed image transmission scheme. The technique used for the efficient Forward error correction technique Punctured Turbo Codes is described in Section 3. Simulation results are reported in Section 4. Finally, conclusion given in Section 5. 


\section{SYSTEM MODEL}

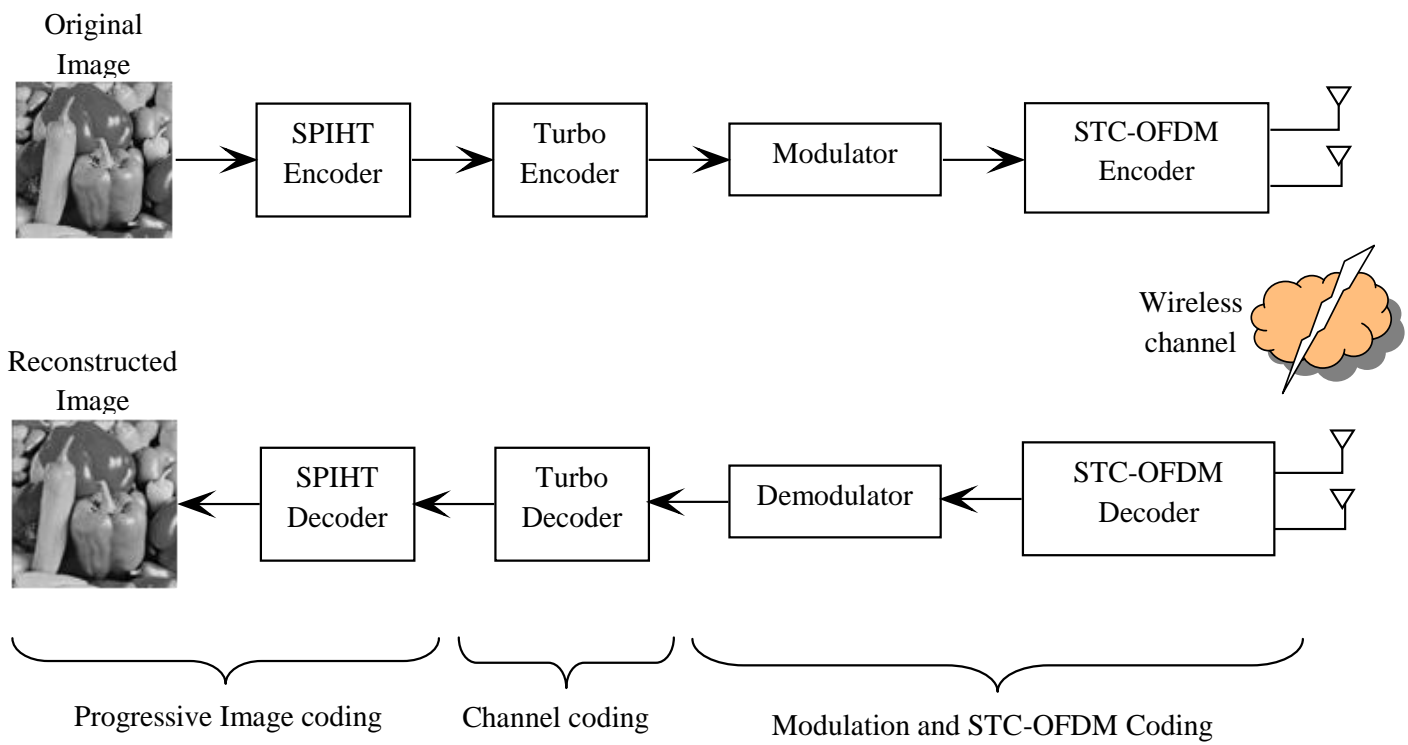

Fig. 1. System structure for Robust Image Transmission over Space Time Coded OFDM systems Using Punctured Turbo Codes.

Here, we propose a PTCS scheme to improve the quality of the SPIHT compressed image transmission by the PTC [16]. By employing iterative decoding principles, turbo codes achieve bit error rates close to the Shannon limit. This powerful forward error correction technique has been applied to SPIHT coding to provide a robust image communication technique. The SPIHT bit stream is packetized into sourcepackets of size $S_{t}$ bits for packet $t, i=1, \ldots, N$. A PTC scheme is used to protect source-packet $t$. The resulted code words are of equal size and treated as OFDM blocks to the transmitter. There are total of $N$ OFDM blocks, where $N$ is decided by the transmission rate. The STBC for two transmit antennas $\left(N_{T}\right)$ is proposed by Alamouti [7]. The maximum-likelihood decoding can achieved for STBC based only on linear processing at the receiver.

The Fig.1 gives the block diagram of the progressive compressed image transmission system. As the output of the SPIHT encoder is progressive, the SPIHT embedded bit stream send to the FEC tech. PTC, resulting bits modulated and transmitted by STC OFDM encoder and passed through a fading channel with Rayleigh characteristics. At the receiver, the received symbols are first decoded by OFDM STC decoder then forwarded to demodulator followed by turbo decoder; at last SPIHT decoder reconstructs the transmitted image. By using OFDM, the whole bandwidth is divided into large number of sub channels, where each sub channel is nonfrequency selective and the inter symbol interference (ISI) can be neglected when guard interval is inserted in each OFDM symbol. In flat Rayleigh fading environment, the received signal model described as

$$
r(t)=h(t) s(t)+n(t)
$$

where $h(t)$ fading channel impulse response, $s(t)$ is the baseband transmitted signal with signal energy $E, n(t)$ is additive white Gaussian noise with variance $N_{0}$.

\section{PUNCTURED TURBO CODING}

\subsection{Turbo Encoder}

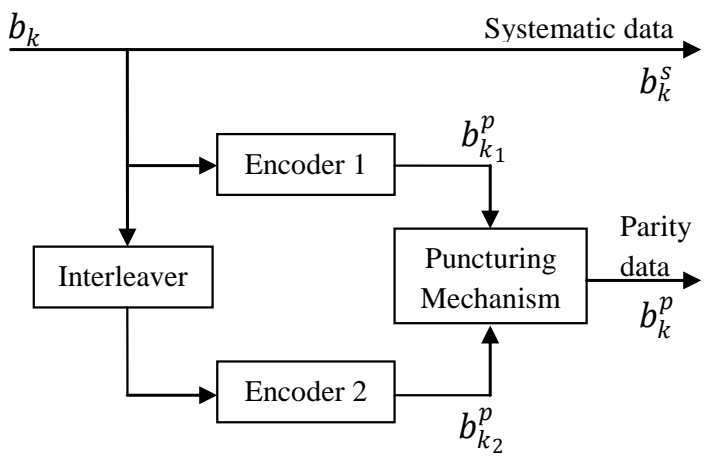

Fig. 2. Punctured Turbo Encoder.

The main procedure of our punctured turbo encoder [13] is shown in Fig.2. There are one interleaver and two Recursive Systematic Convolutional (RSC) encoders. The compatible puncturing mechanism is adopted in the output of the two RSC encoders. The interleaver is to "scramble" bits in a (pseudo) random, albeit predetermined fashion. Suppose the coding rate of the RSC is $1 / 2$, the length is $L$, the memory is $M=K-1$, and the generators of the two Recursive Systematic Convolutional (RSC) encoders are $\mathrm{G}_{1}=\left[g_{10}, g_{11}, \ldots, g_{1, L-1}\right]$ and $\mathrm{G}_{2}=\left[g_{20}, g_{21}, \ldots, g_{2, L-1}\right]$ respectively.

In Fig.2, turbo encoder with rate of $1 / 3$ is shown. Here, $b_{k}^{s}$ is systematic bit, $b_{k_{1}}^{p}$ and $b_{k_{2}}^{p}$ are the parity check bits, respectively. The input binary data sequence $b_{k}=\left(b_{1}, \ldots, b_{n}\right)$ is passed through a RSC encoder 1 , and a coded bit stream, $b_{k_{1}}^{p}$, is formed. The sequence then interleaved in pseudorandom 
manner and passed through RSC encoder 2, and a second coded bit stream, $b_{k_{2}}^{p}$ is generated.

Here, the critical point is, at the decoder side, similar interleaver/de-interleaver blocks are to be repeated. Then the outputs $b_{k_{1}}^{p}$ and $b_{k_{2}}^{p}$ are punctured and the data denoted as $b_{k}^{p}$. To combat bursty errors in fading channels, the employment of a random interleaver is necessary in practical image transmission. Systematic and parity data are modulated, which helps the data passage through the channel with high error performance.

\subsection{Turbo Decoder}

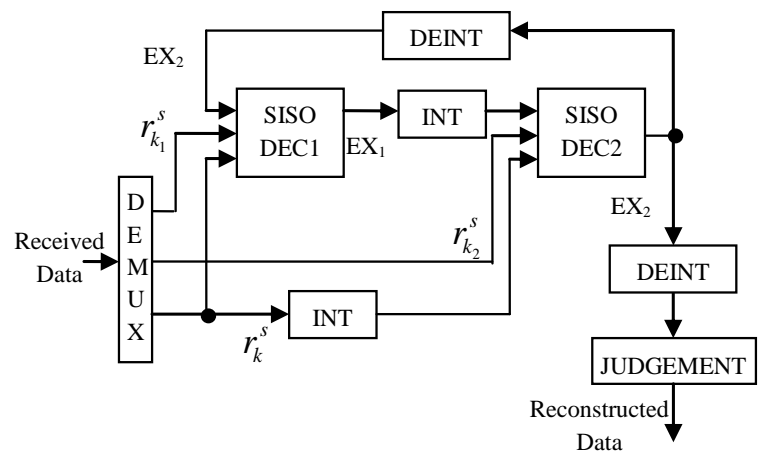

Fig. 3. Punctured Turbo Decoder.

The turbo decoder works in an iterative fashion, as shown in Fig.3. One loop is performed for each iteration [14].As in Fig.3, noisy systematic $r_{k}^{s}$ and noisy parity $r_{k}^{p}$ input sequences are fed into the decoder scheme. After Demultiplexer (DEMUX), the input sequence is passed to two different Soft-Input Soft-Output (SISO) decoder blocks; SISO-DEC1 and SISO-DEC2. ENC1 and ENC2 are decoded sequences formed at the output of SISO-DEC1 and SISODEC2, respectively. All the decoders are based on Maximum APosteriori (MAP) algorithm. SISO-DEC1 decodes noisy inputs; received systematic sequence of soft estimates of the transmitted data bits $b_{k}$ and this output is denoted as extrinsic data $\mathrm{EX}_{1}$.

Since the second SISO-DEC2 inputs are interleaved data, the first decoder output and noisy systematic received value $\left\{r_{k}^{s}\right\}$ are both interleaved. Thus the interleaved systematic received values $\left\{r_{k}^{s}\right\}$ and the sequence of received parity values $\left\{r_{k_{2}}^{p}\right\}$ from the second encoder ENC2 and interleaved extrinsic information $\mathrm{EX}_{1}$ are fed into the second decoder, SISO-DEC2. The output of this decoder are not sufficient in the view of BER performance, then, SISO-DEC2 output is deinterleaved and fed into the first decoder. Thus, the first iteration is processed. The iterations continue until considerable performance is obtained in the decoding stage. The decoders SISO-DEC1 and SISO-DEC2 use the log MAP algorithm to estimate information data. The MAP algorithm estimates a posteriori (APP) of each state transition, information bit produced by Markov process gives the noisy received signal $r$. After calculation of APPs for all possible values of the desired quantity, a hard decision is made by taking the quantity with highest probability. The MAP algorithm calculates the APPs of message bits $P\left[b_{i}=1 \mid r\right]$ and $P\left[b_{i}=0 \mid r\right]$, which are then put into LLR(log likelihood ratio) form according to

$$
\Lambda_{i}=\ln \left\{P_{i}\left[b_{i}=1 \mid r\right]\right\}-\ln \left\{P_{i}\left[b_{i}=0 \mid r\right]\right\}
$$

Here, $b_{i}$ is the message bit associated with the state transitions and $P[$.$] is the conditional probability, (2) is$ according to MAP algorithm. The algorithm first finds the probability of each state transition given the noisy channel observation $y$ as

$$
P\left[x_{i} \rightarrow x_{i+1} \mid r\right]=\frac{P\left[x_{i} \rightarrow x_{i+1}, r\right]}{P[r]}
$$

Where, $x_{i}$ represents the state of the trellis at the time $i$. The conditional probability $P[$.$] \quad is calculated for state$ transition $x_{i}$ to $x_{i+1}$. The numerator rewritten as

$$
P\left[x_{i} \rightarrow x_{i+1}, r\right]=\delta\left(x_{i}\right) \phi\left(x_{i} \rightarrow x_{i+1}\right) \rho\left(x_{i+1}\right)
$$

Here,

$$
\begin{aligned}
& \delta\left(x_{i}\right)=P\left[x_{i},\left(r_{0}, \ldots, r_{i-1}\right)\right] \\
& \phi\left(x_{i} \rightarrow x_{i+1}\right)=P\left[x_{i+1}, r_{i} \mid x_{i}\right] \\
& \rho\left(x_{i+1}\right)=P\left[\left(r_{i+1}, \ldots, r_{L-1}\right) \mid x_{i+1}\right]
\end{aligned}
$$

The term $\phi\left(x_{i} \rightarrow x_{i+1}\right)$ is the branch metric associated with the transition $x_{i}$ to $x_{i+1}$. For log-MAP algorithm

$$
\begin{gathered}
\Lambda_{i}=\ln \left\{\sum_{s_{1}} \exp \left[\delta\left(x_{i}\right)+\not d\left(x_{i} \rightarrow x_{i}+1\right)+\not\left(x_{i+1}\right)\right]\right\}- \\
\ln \left\{\sum_{s_{0}} \exp \left[\delta\left(x_{i}\right)+\not \partial\left(x_{i} \rightarrow x_{i}+1\right)+\not\left(x_{i+1}\right)\right]\right\}
\end{gathered}
$$

Here $X_{1}=\left\{\left(x_{i}=X_{j}\right) \rightarrow\left(x_{i+1}=X_{j}\right): b_{i}=1\right\}$ defines a set of all state transitions for which message bit of " 1 " and in the same manner $X_{0}=\left\{\left(x_{i}=X_{j}\right) \rightarrow\left(x_{i+1}=X_{j}\right): b_{i}=0\right\}$ is the set of all the transitions associated with a message bit is " 0 ", and $\mathscr{\phi}\left(x_{i} \rightarrow x_{i+1}\right)=\ln \phi\left(x_{i} \rightarrow x_{i+1}\right), \quad g\left(x_{i}\right)=\ln \delta\left(x_{i}\right) \quad$ and $g\left(x_{i}\right)=\ln \rho\left(x_{i}\right)$.

In turbo coding, the MAP algorithm makes hard decision on only information bits at the last decoder iteration. The decoder estimates the result according to LLR after several iterations to attain significant performance.

\section{SIMULATION RESULTS}

In this section, we present simulation results for transmitting images over a Rayleigh fading channel. The proposed scheme was experimentally evaluated for the transmission of test images "peppers", "Barbara", "cameraman" and "Lena" over the M-PSK(M-constellation size) modulated STC-OFDM-systems. The test image is compressed using the SPIHT standard to a bit rate of $0.5 \mathrm{bpp}$ 
for all simulations. In SPIHT technique, "bior 4.4" used to get the wavelet coefficients and then the coefficients are processed as per SPIHT algorithm [9] to get embedded bit stream. This image is then encoded by the rate $1 / 2 \mathrm{PTC}$ and modulated, then forwarded to STC-OFDM encoder. Given the set of BER curves in Fig.5, only two bounds, i.e., the lower bound with for $N_{T}=2, N_{R}=1$ and upper bound with $N_{T}=2, N_{R}=2$ are considered. In our simulation, we use a flat fading Rayleigh channel model [13] with Doppler frequency $200 \mathrm{~Hz}$ and delay spread $40 \times 10^{-6} \mathrm{sec}$. The simulation parameters for PTC scheme are, generation matrix is $g=\left[\begin{array}{lllllll}1 & 1 & 1 & 1 & 1 & 0 & 1\end{array}\right]$, logMAP decoding algorithm is considered up to 5 iterations. Tab.I Gives the PSNR $(\mathrm{dB})$ performance of test images which are protected by PTC scheme over various SNR range. For comparison the PCCS system is considered which is similar to the structure as shown in Fig.1, but here we are using PCC as channel coding.

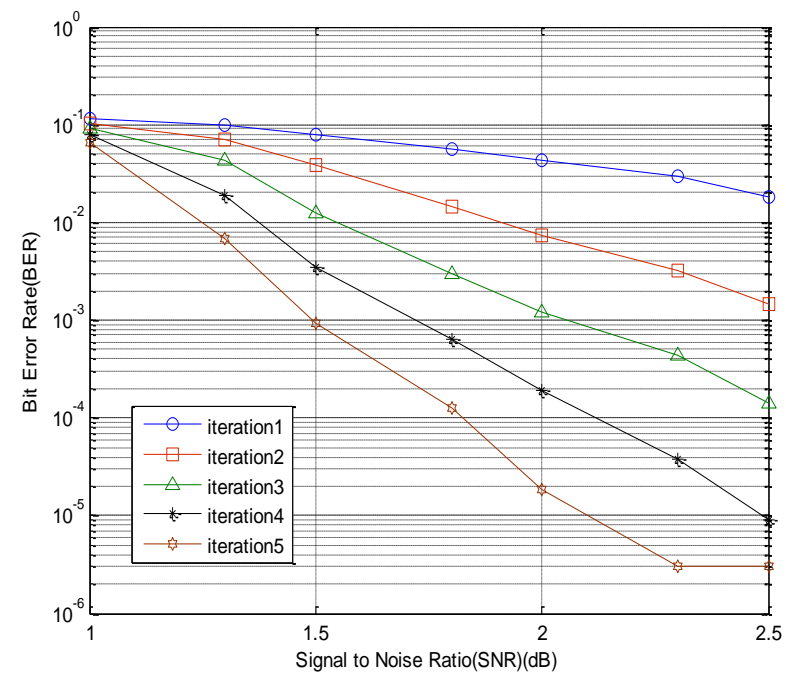

Fig. 4. BER performance curves for various iterations for PTC over Gaussian noisy channel.

The parameters for simulation are, rate of the code is $1 / 2$, constraint length is 7 , generation matrix represented in octal as [171 133], Viterbi decoding considered with hard decision. BER performance of PTC encoder-decoder section for five iterations over Gaussian noisy channel is evaluated and is as shown in Fig 4, the parameters for simulation is same as mentioned above. In this figure, it was shown the when the receiver is equipped to allow a bit complexity by providing more number of iterations, then we can expect a better performance. Here, the receiver with 5 iterations has around a $2 \mathrm{~dB}$ SNR gain compared to the receiver with 1 iteration.

Fig. 5 depicts that the BER performance of PTC based STCOFDM system at different SNR value while varying modulation order. High-order constellations assure more transmitted bits per symbol. In the case of high-order constellations, the points must be closer together and are thus more susceptible to noise and other corruption, which results in a higher BER, and so high-order modulation can deliver more less reliable data than lower-order modulation. From Fig. 5, it is clear that the BER performance for end to end transmission scheme (as shown in Fig.1) is good as compare with PCCS [2].

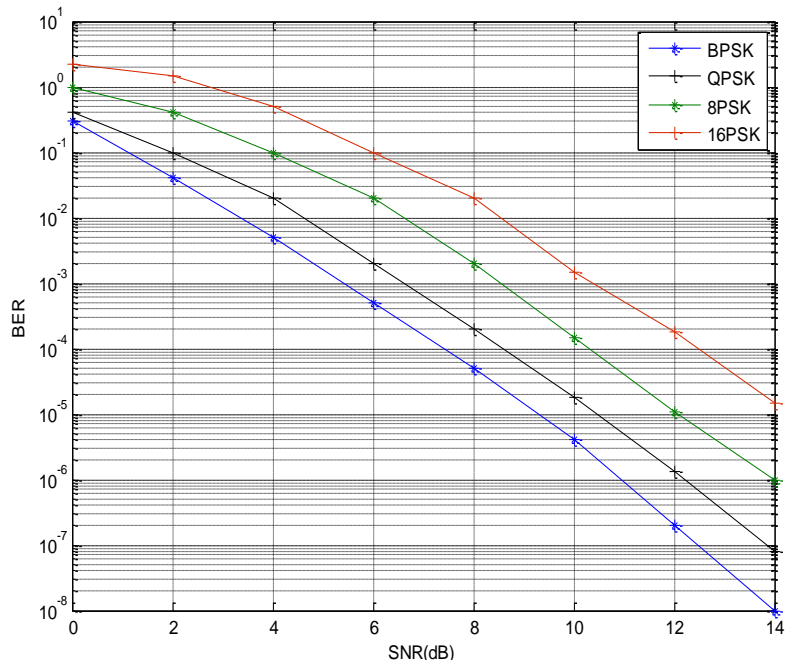

(a)

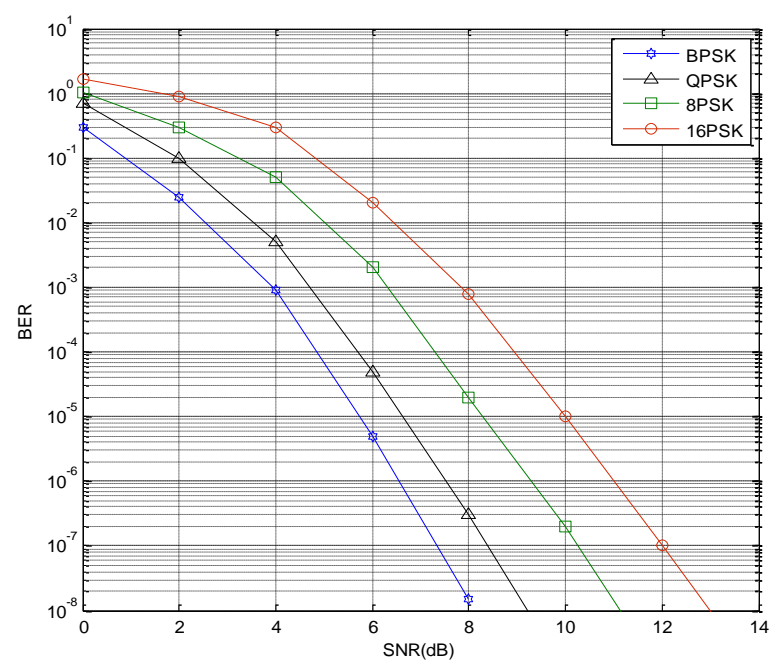

(b)

Fig. 5. Performances of PTC based STC-OFDM systems for various constellation sizes (a) $N_{T}=2, N_{R}=1$, and (b) $N_{T}=2, \mathrm{~N}_{\mathrm{R}}=2$

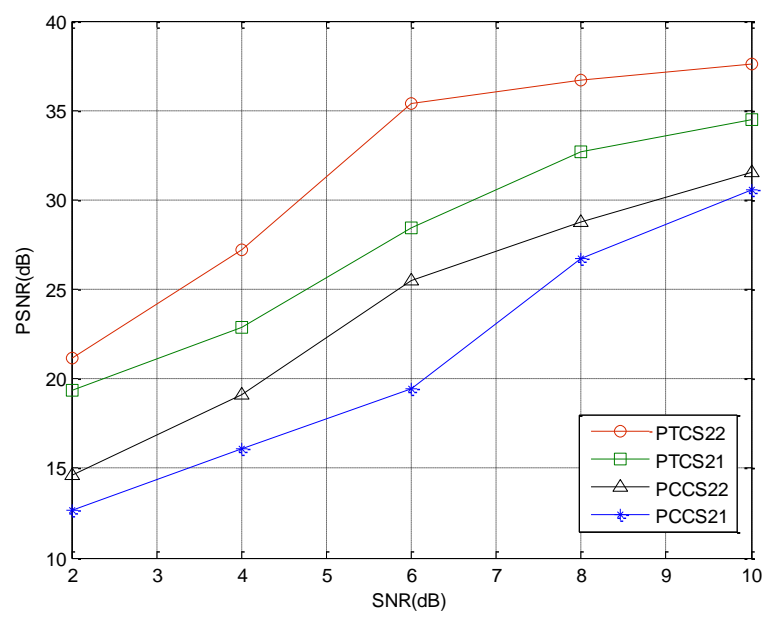

Fig. 6. PSNR performance comparison for proposed scheme and PCCS 
The Fig. 6 shows Peak Signal to Noise Ratio performance with respect to SNR for given range $2 \mathrm{~dB}$ to $10 \mathrm{~dB}$ in which it is very transparent that the PSNR performance for our proposed scheme is quite high compare with PCCS. In figure, PCCS21 represents Punctured Convolutional Coded- SPIHT with $N_{T}=2$ and $N_{R}=1$, similarly PCCS22 represents $N_{T}=2$ and $N_{R}=2$. PTCS21 implies our proposed scheme with $N_{T}=2$ and $N_{R}=1$, PTCS22 gives plot for $N_{T}=2$ and $N_{R}=2$. This image transmission system is evaluated by its PSNR performance. We compute reconstructed image PSNR by

$$
\mathrm{PSNR}=\log _{10}\left(\frac{255^{2}}{\mathrm{MSE}}\right) \mathrm{dB}
$$

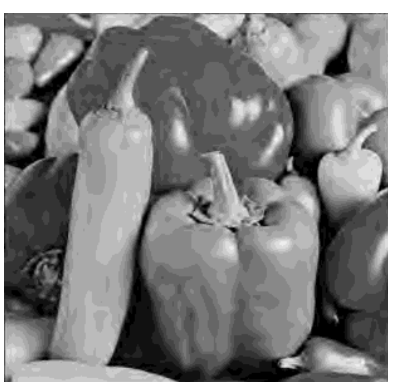

(a)

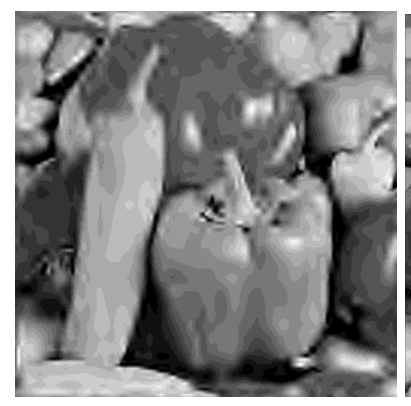

(c)

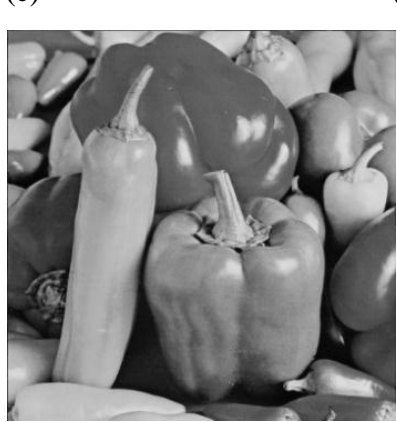

(e)

Fig. 7. Visual comparison for the "Peppers" image using the PCCS and the proposed method. (a) Original (b) $\operatorname{PCCS}\left(N_{T}=2, N_{R}=1\right)(21.19 \mathrm{~dB})(\mathrm{c}) \operatorname{PCCS}\left(N_{T}=2, N_{R}=2\right)$ (24.32 dB) (d) PTCS $\left(N_{T}=2, N_{R}=1\right)(28.71 \mathrm{~dB})$ (e) PTCS $\left(N_{T}=2, N_{R}=2\right)(35.38 \mathrm{~dB})$

We tested the performance of this system for a Rayleigh fading channel with SNR range from $0 \mathrm{~dB}$ to $14 \mathrm{~dB}$. Simulation results after 5 iterations of turbo decoding are shown in fig.5. After the Turbo decoder finished several iterations, the decoded data then forwarded to SPIHT decoder to reconstruct the image. For visual comparison the reconstructed images are shown in Fig. 7 for two bounds of PTCS and also for PCCS. From these figures, we can observe that the proposed PTCS is outperforming the PCCS.

Table 1. PSNR Performance Comparison Heading and text fonts.

\begin{tabular}{|l|c|c|c|}
\hline Image name & SNR=2dB & SNR=4dB & SNR=6dB \\
\hline Lena & $22.33 \mathrm{~dB}$ & $27.82 \mathrm{~dB}$ & $38.71 \mathrm{~dB}$ \\
\hline Barbara & $19.96 \mathrm{~dB}$ & $23.12 \mathrm{~dB}$ & $33.92 \mathrm{~dB}$ \\
\hline Peppers & $21.19 \mathrm{~dB}$ & $27.18 \mathrm{~dB}$ & $35.38 \mathrm{~dB}$ \\
\hline Cameramen & $18.37 \mathrm{~dB}$ & $22.45 \mathrm{~dB}$ & $33.58 \mathrm{~dB}$ \\
\hline
\end{tabular}

Table I gives the PSNR in $\mathrm{dB}$ for the test images "Lena.bmp", "Barbara.png", "Peppers.png", "cameramen.tif" over the given SNR range in $\mathrm{dB}$. It is very transparent that for the test images improvement in PSNR performance quiet high for proposed system as compared to the PCCS over the specified SNR range.

\section{CONCLUSIONS}

A robust end to end progressive compressed image transmission scheme PTCS is described in this paper. In this scheme, the turbo codes fits best to protect SPIHT embedded bit stream. We evaluated the STC-OFDM system by turbo codes with SPIHT for image transmission so as to map the BER performance into expected reconstructed image quality in PSNR. Simulations show that this method provides better BER performance and comparison with PCC and PTC is done. So this system based iterative detection provides an upper bound on the performance of the practical image transmission system. There are several directions in which we could carry on our future work. One direction is to apply better compression standard other than SPIHT.

Another Direction of future research is to make usage of adaptive modulation. An efficient adaptive modulation scheme can be realized by increasing the number of transmit and receive antennas to achieve more diversity gain. But, this method is not feasible when many antennas are not allowed. Another method is to apply other efficient channel code such as low density parity check code to combat fading noise in the STC based OFDM systems.

\section{REFERENCES}

[1] Nikolaos Thomos \& Michael G. Strintzis, "Wireless Image Transmission Using Turbo Codes and Optimal Unequal Error Protection", IEEE transactions on image processing, 14, 1890-1901, 2005.

[2] Yong sun, Zixiang \& xiong, "Progressive Image Transmission over Space Time Coded OFDM Based MIMO Systems with Adaptive Modulation", IEEE Transactions on mobile computing, 5, 1016-1028, 2006.

[3] J. Hagenauer, "Rate Compatible Punctured Convolutional Codes (RCPC Codes) and Their Applications", IEEE Transactions Communications, 36, 389-400. 1988. 
[4] T.V. Ramabadran and S.S.Gaitonde, "A Tutorial on CRC Computations”, IEEE Micro, 8, 62-75. 2002.

[5] Qian Mao \& Boqing Xu, "A New Scheme to Improve the Quality of Compressed Image transmission by Turbo Unequal Error Protection Codes", Conference on Intelligent Information Hiding and Multimedia Signal Processing, 226-229, 2011.

[6] Ming Jiang and Lajos Hanzo, "Multi user MIMO-OFDM for Next Generation Wireless Systems", Proceedings of the IEEE, 95, 1430- 1469. 2007

[7] S.M.Alamouti, "A Simple Transmit Diversity Techniques for Wireless Communications", IEEE Journal of Communications, 16, 1451-1458, 1998.

[8] Ramji Prasad, "OFDM for Wireless Communications Systems”, London, Artech House publications, 2004.

[9] A. Said and W.A. Pearlman, "A New, Fast, and Efficient Image Codec Based on Set Partitioning in Hierarchical Trees", IEEE Transactions on Circuits and Systems for Video Technology, 6, 243-250, 1996.

[10] J. M. Shapiro, "Embedded Image Coding using Zero Trees of Wavelet Coefficients", IEEE Transactions on Signal Processing, 41, 3445-3462, 1993.
[11] H. Bolckei, D.Gesbert, and A.Paulraj, "On the Capacity of OFDM-Based Spatial Multiplexing Systems", IEEE Transactions on Communications, 50, 2002.

[12] P.G. Sherwood and K. Zeger, "Progressive Image Transmission over Memoryless and Fading channels", IEEE Transactions on Communications, 46, 12, 15551559,1998

[13] Eric K. Hall and Stephen G. Wilson, "Design and Analysis of Turbo codes on Rayleigh Fading Channels", IEEE Journal in selected Areas of communications, 16, 160-174, 1998.

[14] C. Berrou, A. Glavieux and P.Thitimajshima, "Near Shannon Limit Error- Correcting and Decoding: Turbo Codes", Proceedings of IEEE international Conference on Communications, 2, 1064-1070, 1993.

[15] L.Yao \& L. Cao, "Turbo Codes Based Image Transmission for Channels with Multiple Types of Distortion", IEEE Transactions on Image processing, 17, 2112-2121, 2008.

[16] Jie Song and K. J. Ray, "Robust Progressive Image Transmission Over OFDM Systems Using Space-Time Block Code", IEEE Transactions on Multimedia, 4, 394406, 2002. 